Serdar Özyurt

Ankara Yıldırım Beyazıt University, sozyurteybu.edu.tr, Ankara-Turkey

DOI $\quad$ http://dx.doi.org/10.12739/NWSA.2018.13.4.1A0420

ORCID ID $0000-0002-9612-6227$

CORRESPONDING AUTHOR Serdar Özyurt

\title{
PERFORMANCE OF ORTHOGONAL FREQUENCY DIVISION MULTIPLEXING WITH SIGNAL SPACE DIVERSITY VIA SUBCARRIER COORDINATE INTERLEAVING OVER NAKAGAMI-M AND RICIAN FADING CHANNELS
}

\begin{abstract}
In this work, orthogonal frequency division multiplexing technique is combined with signal space diversity and error probability performance of the system is inspected for binary phase shift keying modulation over slow frequency-selective Nakagami-m and Rician fading channels. First, appropriate subcarrier coordinate interleaving techniques are obtained based on the number of subcarriers, the number of resolvable multipaths, and the fading parameters. It is shown that appropriate subcarrier coordinate interleaving techniques under some of the investigated scenarios are the same as the ones under the Rayleigh fading scenario. Subsequently, the bit error rate of the studied system is compared with the bit error rate of the original system. Further, it is demonstrated that the considered system provides significant performance gain beyond the original orthogonal frequency division multiplexing technique under different scenarios.
\end{abstract}

Keywords: Orthogonal Frequency Division Multiplexing, Signal Space Diversity, Subcarrier Coordinate Interleaving, Nakagami-M Fading Channel, Rician Fading Channel

\section{INTRODUCTION}

Orthogonal frequency division multiplexing (OFDM) has been included in many contemporary communication standards such as WiFi (IEEE 802.11), WiMax (IEEE 802.16), DAB (Digital Audio Broadcasting), and DVB (Digital Video Broadcasting) [1]. Additionally, as a different form of OFDM, discrete multi-tone transmission technique constitutes a fundamental part of ADSL (Asymmetric Digital Subscriber Line) and VDSL (Very-high-bit-rate Digital subscriber Line) systems. In a wireless communication system, when the channel between the transmitter and receiver is frequency-selective, a complex filter must be applied on the received signal in order to recover the original transmitted signal by means of filtering. The frequency response of the filter must be identical to the inverse of the frequency response of the channel. Depending on how much frequency-selective the channel is, the design and implementation of such a filter may be quite challenging if possible. In OFDM, on the other hand, the frequency-selective channel is divided into numerous subchannels in such a way that all the subchannels can be considered to possess flat frequency responses. By sending data in a low-rate fashion from each subchannel, the communication between the transmitter and receiver is carried out over many parallel subchannels. As all the subchannels exhibit flat

\section{How to Cite:}

Özyurt, S., (2018). Performance of Orthogonal Frequency Division Multiplexing with Signal Space Diversity Via Subcarrier Coordinate Interleaving over Nakagami-M and Rician Fading Channels, Engineering Sciences (NWSAENS), 13(4):291-301, DOI : $10.12739 /$ NWSA.2018.13.4.1A0420. 
frequency responses, the channel equalization at the receiver can be performed by means of a scalar multiplication instead of a complicated filtering operation [2]. Additionally, the fact that Fourier transform included in OFDM can be implemented effectively using the modern baseband techniques has increased the popularity of OFDM. On the other hand, when any of the subchannels experiences a low channel gain, this can cause an incorrect detection on the relevant symbol at the receiver. This poses a serious disadvantage for the original OFDM method.

Signal space diversity (SSD) also known as modulation diversity [3 and 9] is based on utilizing the orthogonal basis in the signal space for the purpose of increasing the diversity order and hence enhancing the error performance of the system. This benefit comes with no increase in bandwidth or power consumption and no significant growth in complexity. For the systems employing two dimensional signal constellations, SSD can be achieved in two steps [3 and 9]. In the first stage, it is ensured that no two symbols in the signal constellation has identical in-phase (I) and quadrature (Q) coordinates. In this way, any symbol can be uniquely identified by either of its two coordinates. The $I$ and $Q$ coordinates of the transmitted symbol are made sure to experience independent fading states in the second step. The first condition mentioned above can be achieved by properly rotating the signal constellation. There exist multiple methods for performing the second step. For instance, this requirement can be satisfied by employing time domain interleaves and deinterleaves at the transmitter and receiver, respectively. However, in such a case, the depth of the employed interleaves must be greater than the coherence time of the channel. This condition can be quite demanding for the transmission of latency-sensitive data. In OFDM system, the second step involved in achieving SSD can also be managed by transmitting the $I$ and $Q$ coordinates of the transmitted symbol over distinct subchannels (subcarrier coordinate interleaving). As it brings about no remarkable extra latency to the original system, this technique is highly desirable as compared to the time domain interleaving scheme.

In this study, OFDM is combined with SSD and the error performance of the system is investigated over slow Nakagami-m and Rician fading channels under binary phase shift keying (BPSK) modulation. In [9], an OFDM/SSD combination based on subcarrier coordinate interleaving has been proposed and analyzed over slow Rayleigh fading channels with BPSK modulation. The scheme in [9] functions based on minimizing the correlation coefficient between the subchannels affecting $I$ and $Q$ coordinates of the transmitted symbol. To this end, several distinct interleaving techniques have been proposed for different cases in [9]. This work assumes Nakagami-m and Rician fading channel scenarios which are more general than the Rayleigh fading scenario (Rayleigh fading is a special case of Nakagami-m and Rician fading scenarios). A comparison with the original OFDM system is provided in terms of the bit error rate (BER) . A literature survey about the inclusion of SSD into OFDM systems can be found in [9].

Throughout the paper, the following notation is utilized: $E\{$.$\} ,$ $\exp (),.||,.(.)^{*}, j, \mathfrak{K}\{$.$\} , and \mathfrak{I}\{$.$\} respectively denote the expected$ value, exponential function, norm for complex numbers (absolute value for real numbers), complex conjugate, $\sqrt{-1}$, real and imaginary parts of a complex number. The probability density function (PDF) of $X$ is represented by $f_{X}(x)$ and $f_{X Y}(x, y)$ stands for the joint PDF of $X$ and $Y$. 
The correlation coefficient between two complex random variables $\mathrm{X}$ and $Y$ is denoted by $\rho_{X, Y}$ and given by

$$
\rho_{X, Y}=\frac{\operatorname{cov}(X, Y)}{\sqrt{\operatorname{var}(X) \operatorname{var}(Y)}}
$$

where $\operatorname{cov}(X, Y)=E\left\{X Y^{*}\right\}-E\{X\} E\left\{Y^{*}\right\}$ is the covariance between the random variables $X$ and $Y$. Additionally, $\operatorname{var}(X)=E\left\{|X|^{2}\right\}-|E\{X\}|^{2}$ stands for the variance of the complex random variable $X$. Two complex random variables $X$ and $Y$ are said to be uncorrelated only if $\operatorname{cov}(X, Y)=0$ and $E\{X Y\}-E\{X\} E\{Y\}=0$, i.e., the pseudo-covariance between $X$ and $Y$ is zero.

\section{RESEARCH SIGNIFICANCE}

This study generalizes the work in [9] by adopting more general fading channel models. Unlike the Rayleigh fading channel assumption in [9], two more comprehensive channel models (Nakagami-m fading and Rician fading channel models) are adopted in this study. It is important to note that Rayleigh fading scenario can be recovered by substituting $m=1$ in Nakagami-m fading case and $K=0$ in Rician fading case. Due to the mentioned reasons, the current work contains original contribution.

\section{SYSTEM MODEL}

It is assumed that a single-antenna transmitter communicates with a single-antenna receiver over a frequency-selective fading channel. Using OFDM, the frequency-selective channel is converted into multiple parallel subchannels each of which experiences flat fading. The communication channel stays constant throughout one OFDM frame and alters from one frame to another in a statistically independent fashion. Full channel state information is available only at the receive side. Assume that the number of subcarriers and the number of resolvable multipaths are respectively represented by $N$ ( $N$ is a power of two and $\mathrm{L}(\mathrm{L} \ll \mathrm{N})$. Each transmitted OFDM frame is composed of $\mathrm{N}$ data symbols and L cyclic prefix symbols. In the original ofDM method, the value of $\mathrm{N}$ is assumed to be known at both ends of the transmission link. The scheme inspected in this work assumes that the values of $N$ and $L$ are both known at the transmitter and receiver. In practice, L takes small integer values and the value of $\mathrm{L}$ can be sent back to the transmitter from the receiver using a low-rate feedback channel. With the successful transmission of each frame, $N$ modulated symbols are conveyed to the receiver. Modulated symbols are produced from a BPSK signal constellation which is rotated by an angle of $\theta$ counterclockwise. Let the modulated symbol sequence and the energy allocated for each bit be denoted by $\left\{s_{1}, s_{2}, \ldots, s_{N}\right\}$ and $E_{b}$, respectively. Because of the energy loss while transmitting the cyclic prefix, one can write $\left|\mathrm{s}_{\mathrm{i}}\right|^{2}=\mathrm{E}_{\mathrm{b}}{ }^{\prime}=\mathrm{E}_{\mathrm{b}}(\mathrm{N} /(\mathrm{N}+\mathrm{L}))$. For any modulated symbol $\mathrm{s}_{\mathrm{k}}, \mathrm{s}_{\mathrm{k}}=\mathrm{s}_{\mathrm{k} I}+j$ s $\mathrm{s}_{\mathrm{k}}$ can be written where $\mathrm{k} \in\{1,2, \ldots, \mathrm{N}\}$. Here, $\mathrm{s}_{\mathrm{k} I}$ and $\mathrm{s}_{\mathrm{k} Q}$ stand for the $I$ and $Q$ coordinates of the symbol $s_{k}$. At the transmitter, coordinate interleaving (CI) is applied on the sequence $\left\{\mathrm{s}_{1}, \mathrm{~s}_{2}, \ldots, \mathrm{s}_{\mathrm{N}}\right\}$ just before the inverse fast Fourier transform block. Likewise, coordinate deinterleaving (CD) is performed just after the fast Fourier transform $(\mathrm{FFT})$ operation at the receive side. Let $\left\{\mathrm{X}_{1}, \mathrm{X}_{2}, \ldots, \mathrm{X}_{\mathrm{N}}\right\}$ denote the sequence obtained after the CI operation at the transmitter. Then, we can write $x_{k}=s_{k I}+j s_{i \ell}$ for $i, k \in\{1,2, \ldots, N\}$ and $i \neq k$. In other words, only Q coordinates are interleaved amongst themselves. Assume that the impulse response of the frequency-selective channel is represented by 
the sequence $\left\{h_{0}, h_{1}, \ldots, h_{L-1}\right\}$. Here, $h_{k}$ for $k \in\{0,1, \ldots, L-1\}$ are independent complex random variables. Further, when written in the polar coordinate system as $h_{k}=\left|h_{k}\right| e^{j \theta_{k}}, \quad\left|h_{k}\right|$ and $\theta_{k}$ are independent random variables. If the output of the FFT operation at the receiver is denoted by $\left\{Y_{1}, Y_{2}, \ldots, Y_{N}\right\}$, then one can write $Y_{k}=H_{k} X_{k}+Z_{k}$ for $k \in\{1,2, \ldots, N\}$. We have

$$
H_{k}=\sum_{i=0}^{L-1} h_{i} \exp \left(-j 2 \pi k \frac{i}{N}\right)
$$

Additionally, $Z_{1}, Z_{2}, \ldots, Z_{N}$ are independent and identically distributed (IID) circularly symmetric complex Gaussian random variables each having a variance of $\mathrm{N}_{0} / 2$ per dimension. These variables represent the additive white Gaussian noise (AWGN) components at the receiver and $\mathrm{N}_{0}$ is the one-sided power spectral density of the AWGN. Moreover, $H_{1}, H_{2}, \ldots, H_{N}$ are identically distributed correlated complex random variables. By performing single-tap equalization on $\mathrm{Y}_{\mathrm{k}}$, we can write $\left(\mathrm{H}_{\mathrm{k}}{ }^{*} /\left|\mathrm{H}_{\mathrm{k}}\right|\right) \mathrm{Y}_{\mathrm{k}}=\left|\mathrm{H}_{\mathrm{k}}\right| \mathrm{X}_{\mathrm{k}}+\hat{\mathrm{Z}}_{\mathrm{k}}$ where $\hat{\mathrm{Z}}_{\mathrm{k}}=\left(\mathrm{H}_{\mathrm{k}}{ }^{*} /\left|\mathrm{H}_{\mathrm{k}}\right|\right) \mathrm{Z}_{\mathrm{k}}$ for $\mathrm{k} \in\{1,2, \ldots, \quad \mathrm{N}\}$. Assume that the $Q$ coordinate of the kth modulated symbol is carried on the th subcarrier. Additionally, let $d_{k}$ be the decision variable used by the maximum likelihood detector for the kth modulated symbol. In this case, after the application of $C D, \quad d_{k}=\mathfrak{R}\left\{Y_{k}\right\}+j \mathfrak{J}\left\{Y_{t}\right\}=\left|H_{k}\right| S_{k I}+j$ $\left|H_{t}\right| s_{k Q}+\check{Z}_{k}$ can be written. Here, $\check{Z}_{k}$ denotes the noise component after the application of CD.

\subsection{Nakagami-m Fading Case}

In the first scenario, it is assumed that the magnitude $\left|h_{k}\right|$ has a Nakagami-m distribution with the following PDF

$$
f_{\left|h_{k}\right|}(r)=2 \frac{m^{m}}{\Gamma(m)} r^{2 m-1} \exp \left(-m r^{2}\right)
$$

for $r \geq 0$ where $m$ represents the shape parameter of the PDF. Also, the average power in the kth multipath (tap) is $E\left\{\left|h_{k}\right|^{2}\right\}=1$, i.e., all the taps have identical average power (uniform power delay profile). When $m$ increases, the severity of the fading decreases. Additionally, $\theta_{\mathrm{k}}$ has a uniform distribution between 0 and $2 \pi$ for any $\mathrm{k}$. We assume that all the taps have the same $m$ value. Under the Nakagami-m fading scenario, the expression in (2) is equal to the sum of L IID Nakagami$\mathrm{m}$ random phase vectors. Using statistical methods, it can be shown that $E\left\{H_{k}\right\}=0$ and $E\left\{\left|H_{k}\right|^{2}\right\}=\sum_{i=0}^{L-1} E\left\{\left|h_{i}\right|^{2}\right\}=L$.

The absolute value of the correlation coefficient (ACC) between the $k$ th and th subchannels $\left(H_{k}\right.$ and $\left.H_{t}\right)$ can be shown to be equal to

$$
\left|\rho_{k t}\right|=\frac{\left|E\left\{H_{k} H_{t}^{*}\right\}-E\left\{H_{k}\right\} E\left\{H_{t}^{*}\right\}\right|}{E\left\{\left|H_{k}\right|^{2}\right\}}=\frac{\left|E\left\{H_{k} H_{t}^{*}\right\}\right|}{L}=\frac{\left|\sum_{a=0}^{L-1} \exp \left(-j 2 \pi a \frac{k-t}{N}\right)\right|}{L}
$$

for $k \in\{1,2, \ldots, N\}$ and $t \in\{1,2, \ldots, N\}$, with $0 \leq\left|\rho_{k t}\right| \leq 1$. By evaluating the sum, we can write

$$
\left|\rho_{k t}\right|=\left|\frac{\sin \left(\pi L \frac{k-t}{N}\right)}{L \sin \left(\pi \frac{k-t}{N}\right)}\right| .
$$

The preceding expression indicates that ACC between any pair of subchannels is independent of $\mathrm{m}$. Also, the equation in (5) is in the same form as the one obtained for the Rayleigh fading scenario in [9]. 
Hence, the CI strategies proposed in [9] for the case of Rayleigh fading can be used for the considered system model in a direct manner. Assuming $\mathrm{N}=512$ and $\mathrm{L}=5$, the $\mathrm{ACC}$ is illustrated in Figure 1 where the ACC is minimized for $(k-t)=205$ with a corresponding ACC of 0.00128 .

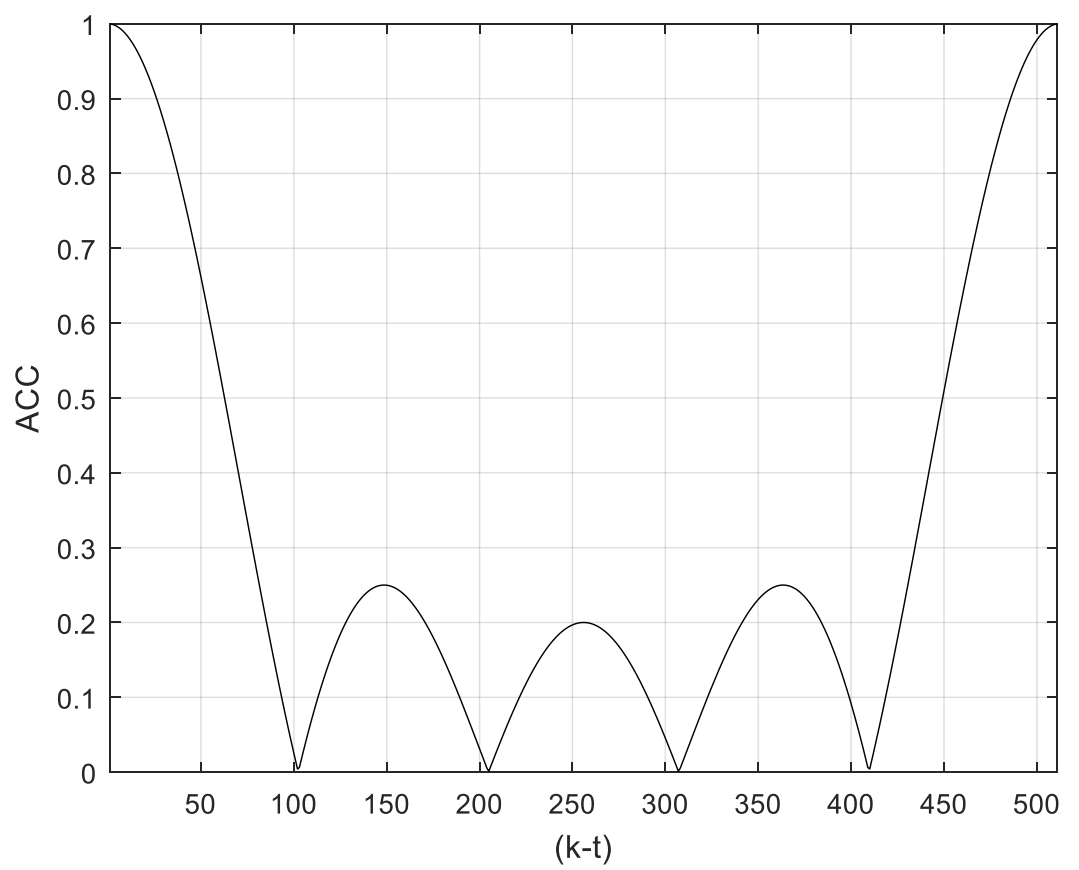

Figure 1. The $\mathrm{ACC}$ for $\mathrm{N}=512$ and $\mathrm{L}=5$ under Nakagami-m fading scenario

\subsection{Rician Fading Case}

We consider Rician fading scenario in this part. To this end, we adopt the model presented by International Telecommunication Union [10]. In this respect, the first tap ho with the shortest path delay includes multiple scattered paths and a specular or line-of-sight component [11]. Therefore, ho is a nonzero-mean complex Gausian random variable, which can also be written as $h_{0}=\sqrt{\frac{K}{K+1}}+\frac{1}{\sqrt{K+1}} \tilde{h}_{0}$ where $\tilde{h}_{0}$ is a zero-mean complex Gaussian random variable and $K$ is the Rician $K-$ factor [2]. All the remaining taps,i.e., $h_{1}, \ldots, h_{L-1}$, are IID zero-mean complex Gaussian random variables, which are also independent from $h_{0}$. The PDFs of $\left|h_{0}\right|$ and $\left|h_{1}\right|$ (or of $\left|h_{2}\right|, \ldots,\left|h_{L-1}\right|$ ) are respectively given by

$$
f_{\left|h_{0}\right|}(r)=2(K+1) r \exp \left(-r^{2}(K+1)-K\right) I_{0}(2 r \sqrt{K(K+1)})
$$

for $r \geq 0$ where $I_{0}($.$) is the modified Bessel function of the first$ kind zero order [12] and

$$
f_{\left|h_{1}\right|}(r)=2 r \exp \left(-r^{2}\right)
$$

for $r \geq 0$. All the multipaths have the same average power as $\mathrm{E}\left\{\left|\mathrm{h}_{\mathrm{k}}\right|^{2}\right\}=1$ for any $\mathrm{k}$. Under the Rician fading scenario, the expression in (2) is equal to the sum of L IID complex Gaussian random variables. Using statistical methods, it can be shown that $E\left\{H_{k}\right\}=\sqrt{\frac{K}{K+1}}$ and $E\left\{\left|H_{k}\right|^{2}\right\}=\frac{K}{K+1}+\frac{1}{K+1}+L-1=L$. 
The ACC between the kth and th subchannels $\left(H_{k}\right.$ and $\left.H_{t}\right)$ can be shown to be

$$
\left|\rho_{k t}\right|=\left|\frac{\sin \left(\pi L \frac{k-t}{N}\right)}{\sin \left(\pi \frac{k-t}{N}\right)} \frac{\exp \left(-j(L-1) \pi \frac{k-t}{N}\right)}{\frac{(L-1) K+L}{K+1}}-\frac{K}{(L-1) K+L}\right|
$$

for $k \in\{1,2, \ldots, N\}, t \in\{1,2, \ldots, N\}$, and $k \neq t$. The ACC between the $k$ th and th subchannels can be calculated efficiently using numerical techniques. In Figure 2, the ACC is plotted for $\mathrm{N}=512, \mathrm{~L}=\{2,4,5\}$, and $K=5$. For $\mathrm{L}=2, \mathrm{~L}=4$, and $\mathrm{L}=5$, the minimum $\mathrm{ACC}$ is obtained at $(\mathrm{k}-\mathrm{t})=256$, $(k-t)=165$, and $(k-t)=124$, respectively. The corresponding ACC values are respectively given by $0.714,0.041$, and 0.024 .

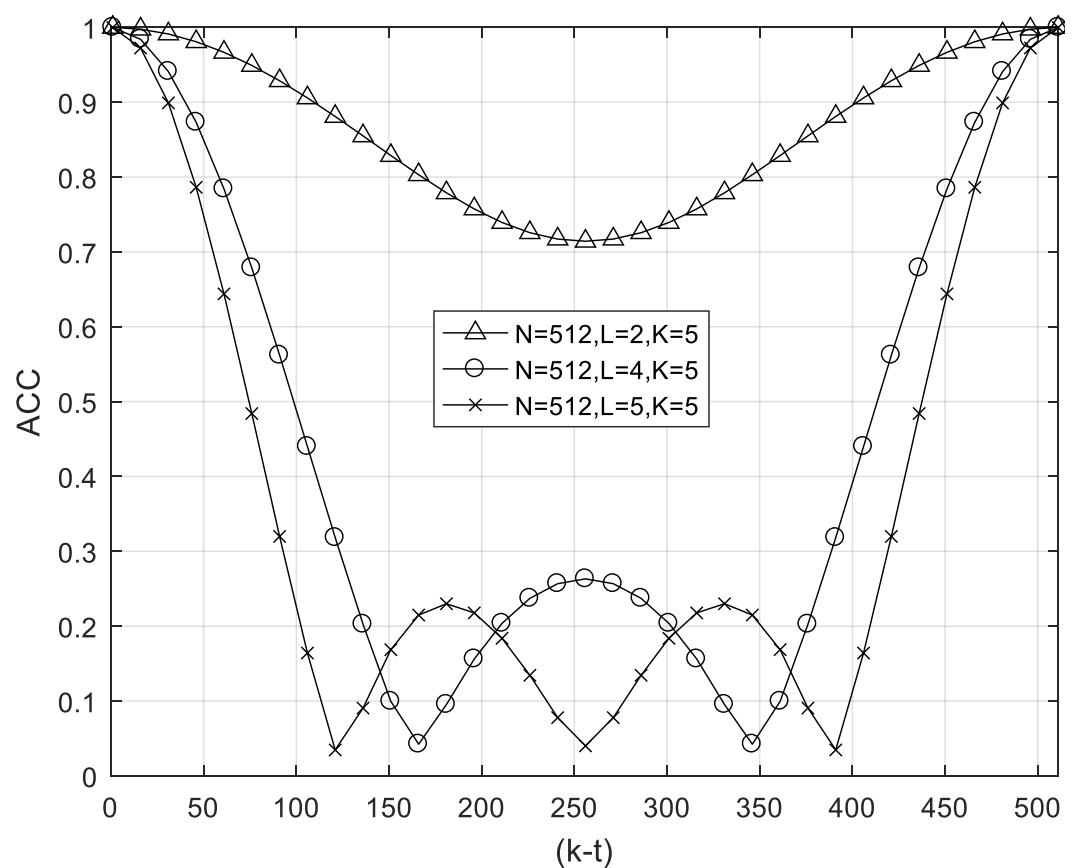

Figure 2. The ACC for $\mathrm{N}=512, \mathrm{~L}=\{2,4,5\}$, and $\mathrm{K}=5$ under Rician fading scenario

\section{COORDINATE INTERLEAVING}

In the sequel, a number of distinct scenarios are studied based on the values of $\mathrm{N}$ and L. Specifically, three cases are investigated as $\mathrm{N}$ and $\mathrm{L}$ have only one common factor, $\mathrm{N}$ and $\mathrm{L}$ have multiple common factors, and $\mathrm{N}$ and $\mathrm{L}$ have no common factor. For each case, suitable CI strategies are presented such that the corresponding ACC expressions are minimized.

\subsection{Scenario 1: $\mathrm{N}=512, \mathrm{~L}=2$}

In this case, (5) becomes zero only for one $(k-t)$ value $(k-t=256)$ under Nakagami-m fading. For the Nakagami-m fading case, the following CI strategy is applied at the transmitter:

$$
X_{k}=\left\{\begin{array}{lr}
\mathfrak{R}\left\{s_{k}\right\}+j \mathfrak{J}\left\{s_{k+256}\right\}, & 1 \leq k \leq 256, \\
\mathfrak{R}\left\{s_{k}\right\}+j \mathfrak{J}\left\{s_{k-256}\right\}, & 257 \leq k \leq 512 .
\end{array}\right.
$$

For the Rician fading scenario, the ACC in (8) is minimized when $(k-t)=256$ for both $\mathrm{K}=3$ and $\mathrm{K}=5$ cases. Consequently, the CI strategy in (9) is also exploited under Rician fading with $K=3$ and $K=5$. 


\subsection{Scenario 2: $\mathrm{N}=512, \mathrm{~L}=4$}

Under this scenario, there exist three distinct $(k-t)$ values $(k-t \in\{128,256,384\})$ for which (5) becomes zero under Nakagami-m fading. All of these values yield identical error performances as the $I$ and $Q$ coordinates of the transmitted symbol experience independent fading with the all three values. For the Nakagami-m fading case, the CI strategy in (9) is utilized. For the Rician fading scenario, the ACC in (8) is minimized when $(k-t)=351$ and $(k-t)=165$ for $\mathrm{K}=3$ and $\mathrm{K}=5$, respectively. Hence, for $K=3$ and $K=5$, the following CI strategies are respectively used at the transmitter:

$$
X_{k}=\left\{\begin{array}{lr}
\Re\left\{s_{k}\right\}+j \Im\left\{s_{k+351}\right\}, & 1 \leq k \leq 161, \\
\Re\left\{s_{k}\right\}+j \Im\left\{s_{k-161}\right\}, & 162 \leq k \leq 512,
\end{array}\right.
$$

and

$$
X_{k}=\left\{\begin{array}{lr}
\Re\left\{s_{k}\right\}+j \Im\left\{s_{k+165}\right\}, & 1 \leq k \leq 347, \\
\mathfrak{R}\left\{s_{k}\right\}+j \mathfrak{J}\left\{s_{k-347}\right\}, & 348 \leq k \leq 512 .
\end{array}\right.
$$

\subsection{Scenario 3: $\mathrm{N}=512, \mathrm{~L}=5$}

Since $\mathrm{N}$ and $\mathrm{L}$ are relatively prime, no (k-t) value can make (5) zero under Nakagami-m fading. The value of (k-t) that minimizes (5) can be found as $(k-t)=205$ with a corresponding ACC of 0.00128 using numerical techniques. Therefore, for the Nakagami-m fading case, the following CI strategy is utilized:

$$
X_{k}=\left\{\begin{array}{lr}
\mathfrak{R}\left\{s_{k}\right\}+j \Im\left\{s_{k+205}\right\}, & 1 \leq k \leq 307, \\
\mathfrak{R}\left\{s_{k}\right\}+j \mathfrak{\Im}\left\{s_{k-307}\right\}, & 308 \leq k \leq 512 .
\end{array}\right.
$$

For the Rician fading scenario, the ACC in (8) is minimized when $(k-t)=121$ and $(k-t)=124$ for $\mathrm{K}=3$ and $\mathrm{K}=5$, respectively. Therefore, for $\mathrm{K}=3$ and $\mathrm{K}=5$, the following $\mathrm{CI}$ strategies are respectively applied at the transmitter:

$$
X_{k}=\left\{\begin{array}{lr}
\mathfrak{R}\left\{s_{k}\right\}+j \mathfrak{I}\left\{s_{k+121}\right\}, & 1 \leq k \leq 391, \\
\mathfrak{R}\left\{s_{k}\right\}+j \mathfrak{I}\left\{s_{k-391}\right\}, & 392 \leq k \leq 512,
\end{array}\right.
$$

and

$$
X_{k}=\left\{\begin{array}{lr}
\Re\left\{s_{k}\right\}+j \Im\left\{s_{k+124}\right\}, & 1 \leq k \leq 388, \\
\Re\left\{s_{k}\right\}+j \Im \mathfrak{J}\left\{s_{k-388}\right\}, & 389 \leq k \leq 512 .
\end{array}\right.
$$

\section{NUMERICAL RESULTS}

In this part, a number of numerical results are illustrated. The inspected system "OFDM with SSD" is compared with the original OFDM approach without SSD "OFDM with no SSD" in terms of the BER. In all figures, $\mathrm{N}=512$ is used. In Figures 3,4, and 5, the Nakagami-m fading scenario is considered whereas the Rician fading case is investigated in Figures 6,7, and 8. In Figure 3, the relevant schemes are compared for $\mathrm{L}=2$ and $\mathrm{m}=\{2,3\}$. The comparison results indicate that "OFDM with SSD" yields considerable SNR gains beyond the original approach "OFDM with no SSD". For instance, $14.3 \mathrm{~dB}$ and $15.7 \mathrm{~dB}$ SNR gains are respectively obtained for $\mathrm{m}=2$ and $\mathrm{m}=3$ at a $\mathrm{BER}$ of $10^{-3}$. The two methods are compared in Figure 4 by adopting $L=4$ and $m=\{2,3\}$. Similar to the preceding results, for a BER of $10^{-4}$, "OFDM with SSD" respectively achieves $14.5 \mathrm{~dB}$ and $13.9 \mathrm{~dB}$ SNR gains for $\mathrm{m}=2$ and $\mathrm{m}=3$ as compared to "OFDM with no SSD". The two techniques are compared in Figure 5 for $\mathrm{L}=5$ and $\mathrm{m}=\{2,3\}$. Under this scenario, as stated before, (5) cannot be made equal to zero for any (k-t) value. Hence, the CI strategy in (12) 
that minimizes (5) is used for this case. In this case, "OFDM with SSD" accomplishes $15 \mathrm{~dB}$ SNR gain at a BER of $1^{-4}$ as compared to "OFDM with no SSD" for both $\mathrm{m}=2$ and $\mathrm{m}=3$. Assuming $\mathrm{L}=2$, the two schemes are compared in Figure 6 for $K=3$ and $K=5$. For a BER of $2 \times 10^{-4}$, "OFDM with $S_{S D}$ " attains $15.1 \mathrm{~dB}$ and $16.3 \mathrm{~dB}$ SNR gains, respectively for $\mathrm{K}=3$ and $K=5$, as compared to "OFDM with no SSD". In Figures 7 and 8 , the BER performances of the two techniques are illustrated using $\mathrm{L}=4$ and $\mathrm{L}=5$, respectively. When $\mathrm{L}=4$ or $\mathrm{L}=5$, "OFDM with $\mathrm{SSD}$ " has around $14.7 \mathrm{~dB} \mathrm{SNR}$ gain beyond "OFDM with no SSD" for both $K=3$ and $K=5$ at a BER of $10^{-4}$.

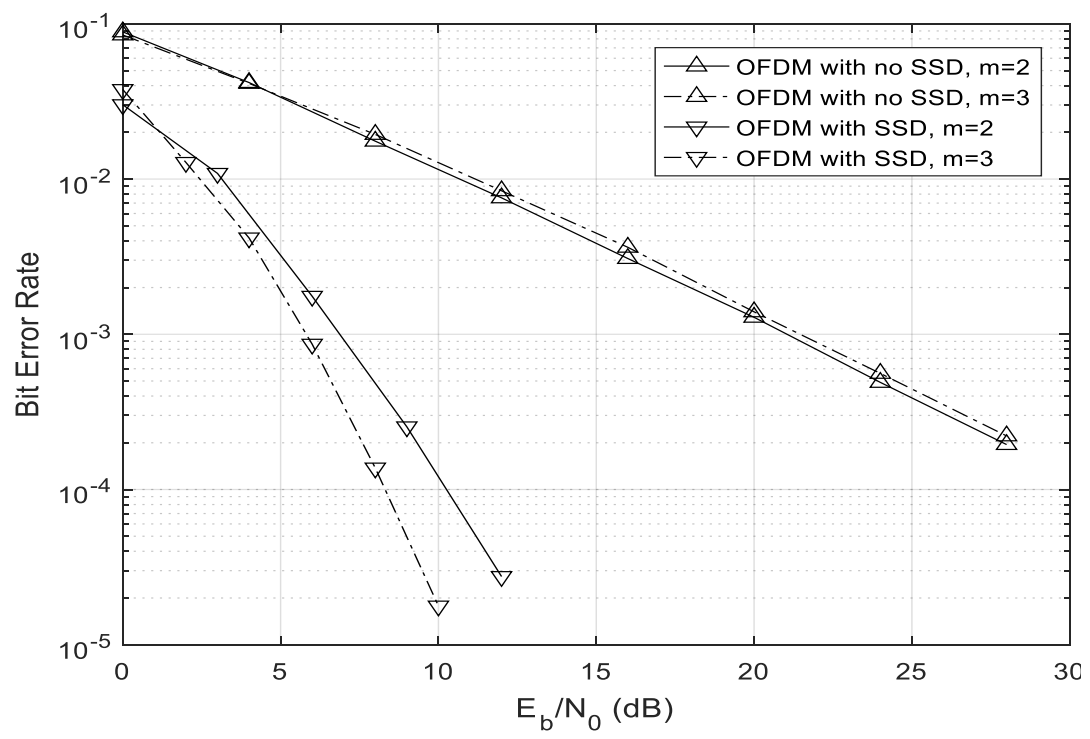

Figure 3. Comparison of the relative techniques over Nakagami-m fading channels for $\mathrm{N}=512$ and $\mathrm{L}=2$

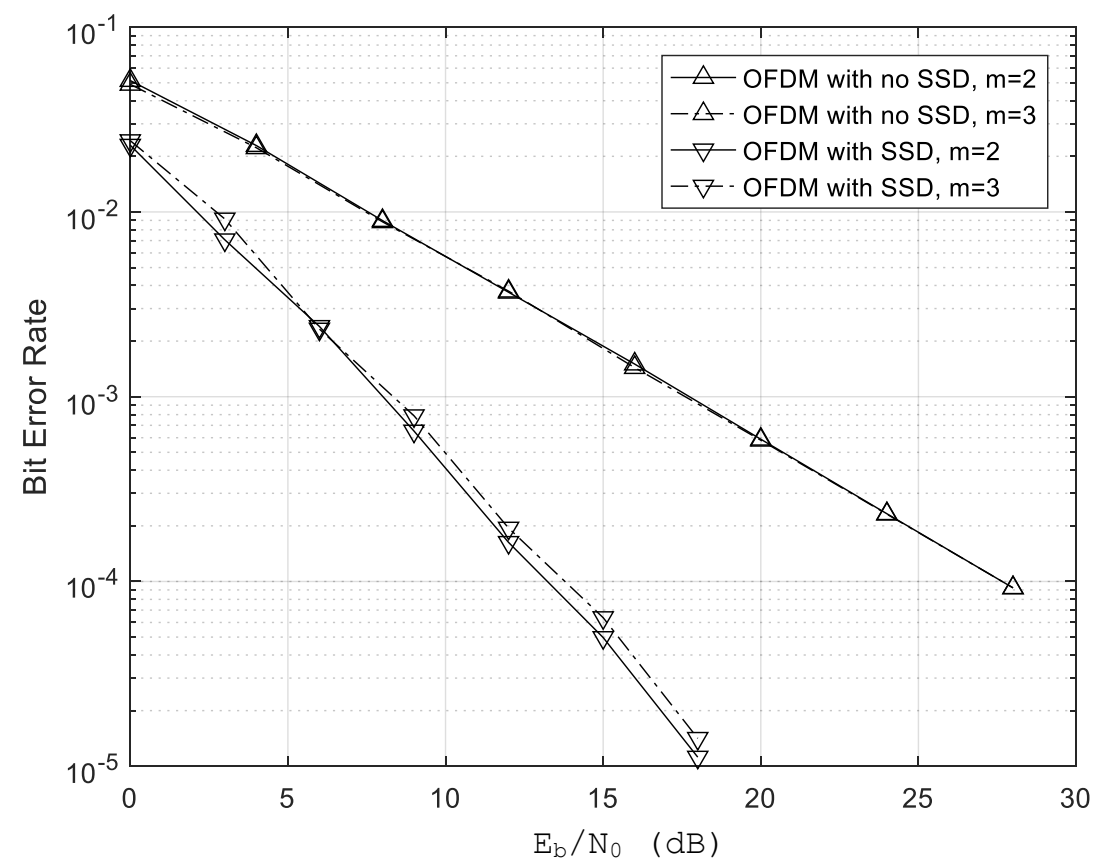

Figure 4. Comparison of the relative techniques over Nakagami-m fading channels for $\mathrm{N}=512$ and $\mathrm{L}=4$ 


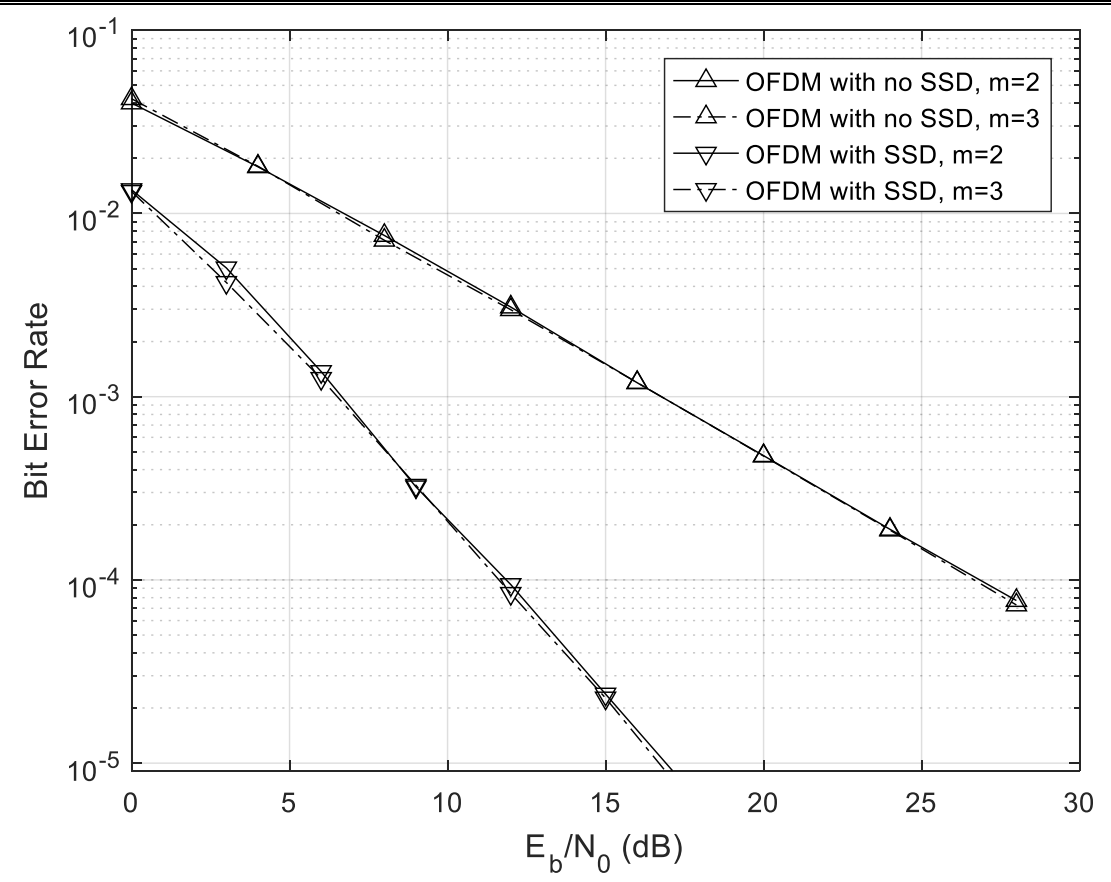

Figure 5. Comparison of the relative techniques over Nakagami-m fading channels for $\mathrm{N}=512$ and $\mathrm{L}=5$

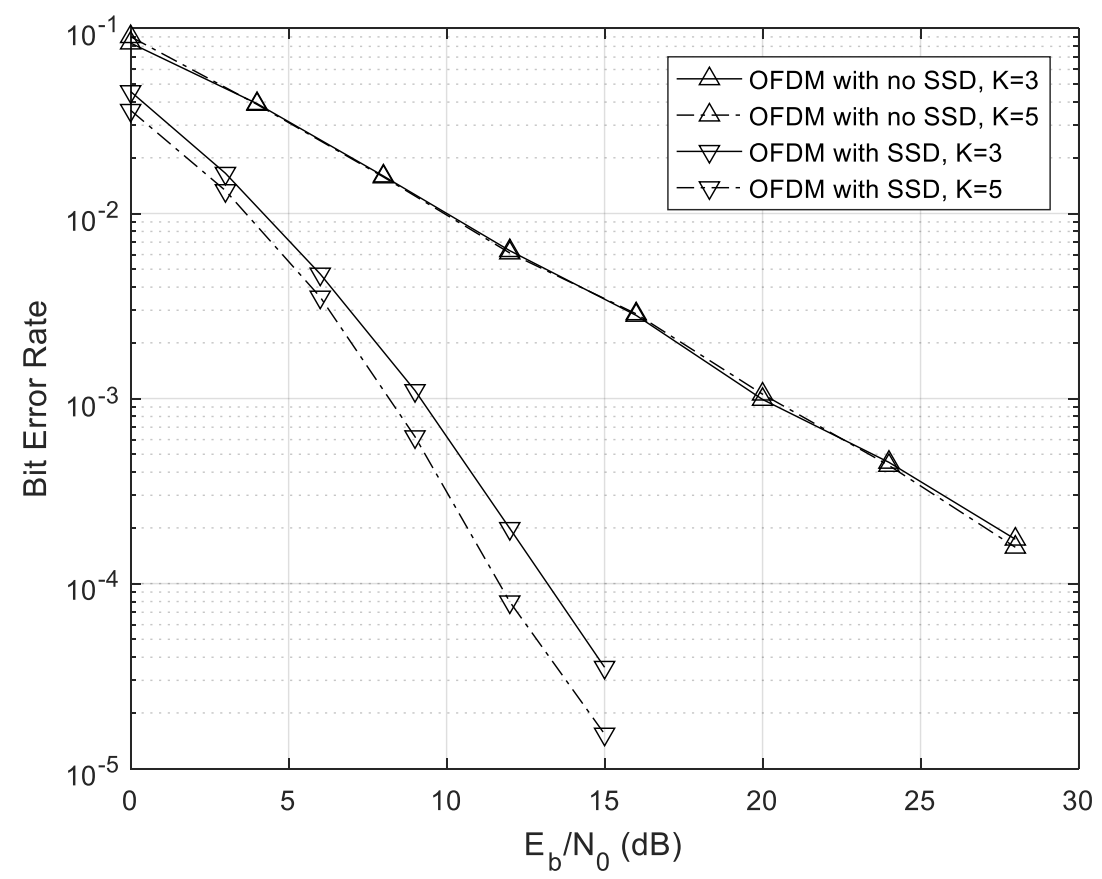

Figure 6. Comparison of the relative techniques over Rician fading channels for $\mathrm{N}=512$ and $\mathrm{L}=2$ 


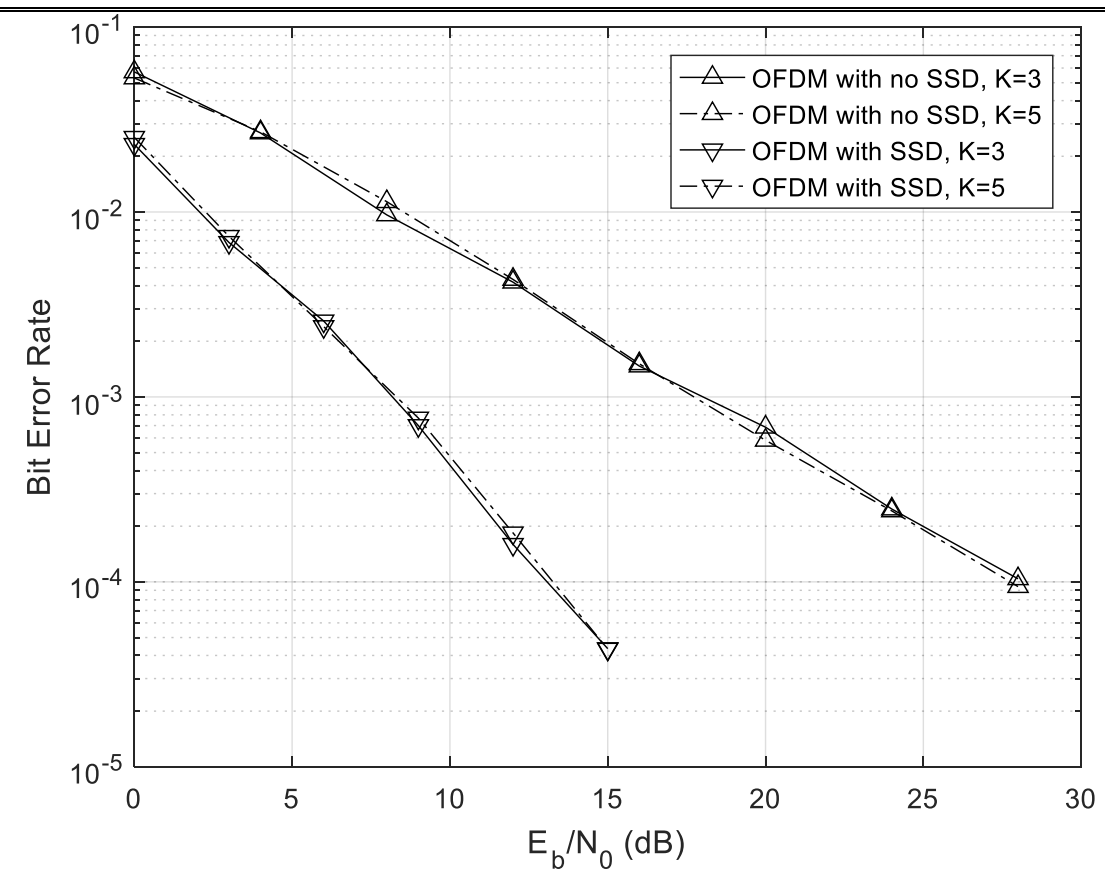

Figure 7. Comparison of the relative techniques over Rician fading channels for $\mathrm{N}=512$ and $\mathrm{L}=4$

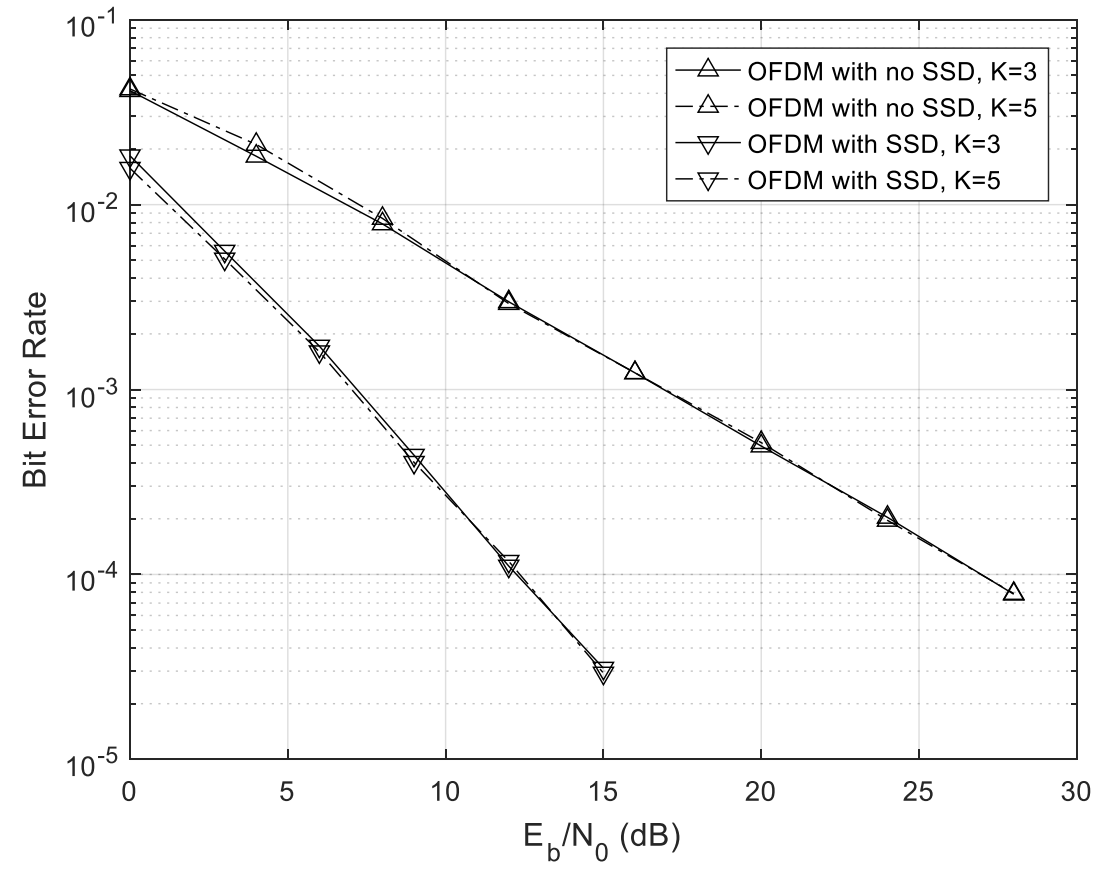

Figure 8. Comparison of the relative techniques over Rician fading channels for $\mathrm{N}=512$ and $\mathrm{L}=5$

\section{CONCLUSION}

The BER performance of an OFDM system combined with SSD has been investigated with BPSK modulation over slow frequency-selective Nakagami-m and Rician fading channels. To this end, with the purpose of enhancing the BER performance of the system, different CI techniques have been studied under distinct scenarios. It has been shown that under Nakagami-m fading, the CI strategies offered for the Rayleigh fading scenario can directly be used in order to have 
indepedendent subchannels affecting the $I$ and $Q$ coordinates of any transmitted symbol. Furthermore, for the Rician fading scenario, the ACC for any pair of subchannels has been expressed in a simple form that can easily be evaluated resorting to numerical techniques. Based on this result, appropriate CI strategies have been presented for the Rician fading case. Additionally, it has been demonstrated that the studied approach yields serious performance gains beyond the original OFDM technique for different fading scenarios. This benefit is acquired without a notable increase in the system complexity. The CI strategies presented in this work can also be used with other twodimensional modulation methods such as quadrature PSK and 8-PSK.

\section{REFERENCES}

[1] Hwang, T., Yang, C., Wu, G., Li, S., and Li, G.Y., (2009). OFDM and its Wireless Applications: A Survey, IEEE Trans. Veh. Technol., Vol:58, No:4, pp:1673-1694.

[2] Tse, D. and Viswanath, P., (2005). Fundamentals of Wireless Communications, Cambridge University Press.

[3] Boulle, K. and Belfiore, J.C., (1992). Modulation Scheme Designed for Rayleigh Fading Channel, in Proc. 26th Annual Conf. Inf. Sciences Sys., Princeton, US.

[4] Taricco, G. and Viterbo, E., (1996). Performance of Component Interleaved Signal Sets for Fading Channels, Electron. Lett., Vol:32, No:13, pp:1170-1172.

[5] Boutros, J. and Viterbo, E., (1998). Signal Space Diversity: a Power- and Bandwidth-Efficient Diversity Technique for the Rayleigh Fading Channel, IEEE Trans. Inf. Theory, Vol:44, No:4, pp: 1453-1467.

[6] Özyurt, S., Kucur, O., and Altunbaş, İ., (2007). Error Performance of Rotated Phase Shift Keying Modulation over Fading Channels, J. of Wireless Personal Commun., Vol:43, No:4, pp: $1453-1463$.

[7] Yllmaz, A. and Kucur, O., (2011). Performance of Rotated PSK Modulation in Nakagami-m Fading Channels, J. of Digital Signal Process., Vol:21, No:2, pp:296-306.

[8] Slimane, S.B., (1998). An Improved PSK Scheme for Fading Channels, IEEE Trans. Veh. Technol., Vol:47, No:2, pp:703-710.

[9] Özyurt, S. and Kucur, O., (2018). Performance of OFDM with Signal Space Diversity Using Subcarrier Coordinate Interleaving, IEEE Trans. Veh. Technol., Accepted, Available Online.

[10] Guidelines for Evaluation of Radio Transmission Technologies for IMT-2000, (1997). Recommendation ITU-R M.1225.

[11] Rainfield, Y.Y., Hong-Yu, L., and Wei, K.T., (2008). QAM Symbol Error Rate in OFDM Systems Over Frequency-Selective Fast RiceanFading Channels, IEEE Trans. Veh. Technol., Vol:57, No:2, pp: $1322-1325$.

[12] Abramowitz, M. and Stegun, I.A., (1972). Handbook of Mathematical Functions with Formulas, Graphs, and Mathematical Tables, Dover Press. 